\title{
A Study of Deformations in a Thermoelastic Dipolar Body with Voids
}

\author{
Marin Marin $1, * \mathbb{*}$, Ibrahim Abbas ${ }^{2}$, Sorin Vlase ${ }^{3} \mathbb{D}$ and Eduard M. Craciun ${ }^{4}$ \\ 1 Department of Mathematics and Computer Science, Transilvania University of Brasov, \\ 500093 Brasov, Romania \\ 2 Department of Mathematics, Faculty of Science, Sohag University, Sohag 82524, Egypt; ibrabbas7@gmail.com \\ 3 Department of Mechanical Engineering, Transilvania University of Brasov, 500093 Brasov, Romania; \\ svlase@unitbv.ro \\ 4 Faculty of Mechanical, Industrial and Maritime Engineering, Ovidius University of Constanta, \\ 900527 Constanta, Romania; mcraciun@univ-ovidius.ro \\ * Correspondence: m.marin@unitbv.ro
}

Received: 5 January 2020; Accepted: 31 January 2020; Published: 9 February 2020

\begin{abstract}
In this paper, we consider the mixed initial boundary value problem in the context of a thermoelastic porous body having a dipolar structure. We intend to analyze the rate of decay of solutions to this problem to ensure that in a finite time, they become null. In our main result, we find that the combined contribution of the dipolar constitution of the body together with voids dissipation and thermal behavior cannot cause vanishing of the deformations in a finite time.
\end{abstract}

Keywords: body with dipolar structure; decay of solutions; localization in time of solution; combined effects; vanishing of deformations

\section{Introduction}

We must outline that our study is dedicated to the dipolar structure for a porous thermoelastic body. The unanimous opinion of the specialists is that the porous media with a dipolar structure very accurately shapes the structure and behavior of the bones of humans and, obviously, of animals.

This kind of material is an integral part of a more general theory, namely the microstructure, whose initiator was Eringen (see, for instance, [1,2]). The utility of the dipolar bodies can be observed from the great number of appeared papers in this subject, see for instance [3-7]. Therefore, our present study is a continuation of research in this domain.

Another kind of the microstructure is also obtained by considering the pores (voids) in the materials, and this was starting by the paper [8] of Nunziato and Cowin. Then, the number of papers with this subject has become impressive. Here, we mention some of these [9-16]. In the opinion of many researchers, the backward in time problem and the problem of localization in a finite time of solutions have many points in common. It is considered that the initiator of the backward in time problems is Serrin which approached this kind of problem by taking into account the Navier-Stokes Equations (see [17]). Some results of uniqueness for the forward in time problem can be found in the study [17]. Recently, the number of articles having as a subject the problem of backward in time has increased greatly. Therefore, we can recommend the work in [18-27]. As a particular observation, the results of Quintanilla in [25] improves the studies of Ciarletta [23] and Ciarletta and Chirita [24]. Other studies of Quintanilla solve some questions regarding the location in time considering the solutions for problems back in time even in the case of theory of thermoelastic bodies with voids and of the theory of Green and Naghdi for thermoelasticity [26,27]. Iovane and Passarella in [28] approached also the elastic bodies with voids. Passarella and Tibullo consider in [29] the problem of backward in time for the thermo-microstretch elastic bodies. Similar results can be found in $[30,31]$. 
We should point out that our idea to consider the problem of localization in a finite time of solutions in the case of the theory of dipolar bodies with voids was inspired by the paper [32] of Quintanilla and Straughan.

The structure of our paper is the following.

In Section 2, we write the system of the differential equations that govern the evolution of our body. After that, as usual, we add the initial conditions and, to complete the mixed problem, we consider the boundary data. Then, we continue with few auxiliary results, which offer some estimations regarding the displacements, the displacements of dipolar type and regarding the function of voids. The main result of our work is regarding the localization in time of solution four our mixed problem. In fact, we prove that is not possible to locate a finite time for vanishing of the deformations.

\section{Preliminaries}

As already seen, our study is dedicated to a thermoelastic dipolar body with voids. We consider the general of an anisotropic and non-homogeneous body, which occupies a regular region $D$, included in the Euclidean space $R^{3}$. The border of the domain $D$ is a regular surface, denoted by $\partial D$. As usual, we use the notation $\bar{D}$ for the closure of the domain $D$, and we have $\bar{D}=D \cup \partial D$. We will suppose that the vectors and tensors have components with indices over 1,2, and 3 . The convention for summation in the case of repeated index is used and the derivation operations are corresponding defined. Therefore, a partial derivative with respect to a spatial coordinate is designates by a subscript preceded by a comma. A superposed dot is used to designate the derivative with regards to $t$, the time time variable. All functions used in our study are supposed be sufficient regular as necessary. Sometimes, the dependence of functions regarding the time or spatial independent variables is omitted, of course, when is no possibility of confusion.

To describe the evolution of a dipolar thermoelastic body, we will use the following variables,

$$
u_{i}(t, x), \phi_{i j}(t, x), \theta(t, x), \varphi(t, x),
$$

where we denoted by $u=\left(u_{i}\right)$ the vector field for displacement, and by $\phi=\left(\phi_{i j}\right)$ the tensor field for dipolar displacement. Also, here, $\theta$ is the temperature variation and $\varphi$ is a function for the volume distribution.

All these functions are defined for $(t, x) \in\left[0, t_{0}\right) \times D$.

Using the above variables $u_{i}(x, t), \phi_{i j}(x, t)$, we will introduce the components of the tensors of strain, namely, $e_{i j}, v_{i j}$ and $\chi_{i j k}$, as follows,

$$
2 e_{i j}=u_{j, i}+u_{i, j}, v_{i j}=u_{j, i}-\phi_{i j}, \chi_{i j k}=\phi_{j k, i} .
$$

We must specify that our further considerations refers to linear theory as such we can consider the Helmholtz's free energy as a quadratic form, regarding to all its independent variables. Let us denote by $W$ the Helmholtz's free density of energy in the initial configuration. As a consequence, in accordance with the principle of conservation of energy, we will develop the function $W$ in series and we retain only the terms of first order and of second order. If we take into account that the initial state (the reference state) is supposed free of charges, we are led to the conclusion that the energy density of Helmholtz has the following expression,

$$
\begin{aligned}
W=\frac{1}{2} A_{i j m n} e_{i j} e_{m n}+D_{i j m n} e_{i j} v_{m n}+F_{i j m n r} e_{i j} \chi_{m n r}+\frac{1}{2} B_{i j m n} v_{i j} v_{m n} \\
+G_{i j m n r} v_{i j} \chi_{m n r}+\frac{1}{2} C_{i j k m n r} \chi_{i j k} \chi_{m n r}+a_{i j} e_{i j} \theta+b_{i j} v_{i j} \theta+c_{i j k} \chi_{i j k} \theta- \\
\quad-\frac{1}{2} c \theta^{2}-m \theta \varphi+\frac{1}{2} A_{i j} \varphi_{, i} \varphi_{, j}+\alpha_{i j} e_{i j} \varphi+\beta_{i j} v_{i j} \varphi+\delta_{i j k} \chi_{i j k} \varphi+\frac{1}{2} \xi \varphi^{2} .
\end{aligned}
$$


If we substitute the Helmholtz's energy in the entropy production inequality, we obtain a relation from where the equations of motion are deduced. Also, the same relation can be used to deduce the constitutive equations. As is known, the constitutive equations give the expression of stress tensors as functions of the strain tensors and some constants of the material. In what follows, we will use the notations $t_{i j}, \sigma_{i j}$ and $\eta_{i j k}$ for the stress tensors. As such, the connections between the tensors $t_{i j}, \sigma_{i j}, \eta_{i j k}$ and the tensors $e_{i j}, v_{i j}, \chi_{i j k}$ are the constitutive equations

Inspired by the procedure used by Green and Rivlin in the paper [6], we adopt a similar technique, so that by taking into account the Helmholtz's energy (3) we are led to the following constitutive equations,

$$
\begin{gathered}
t_{i j}=\frac{\partial W}{\partial e_{i j}}=A_{i j m n} e_{m n}+D_{m n i j} v_{m n}+F_{m n r i j} \chi_{m n r}+a_{i j} \theta+\alpha_{i j} \varphi, \\
\sigma_{i j}=\frac{\partial W}{\partial v_{i j}}=D_{i j m n} e_{m n}+B_{i j m n} v_{m n}+G_{i j m n r} \chi_{m n r}+b_{i j} \theta+\beta_{i j} \varphi, \\
\eta_{i j k}=\frac{\partial W}{\partial \chi_{i j k}}=F_{i j k m n} e_{m n}+G_{m n i j k} v_{m n}+C_{i j k m n r} \chi_{m n r}+c_{i j k} \theta+\delta_{i j} \varphi, \\
h_{i}=\frac{\partial W}{\partial \varphi_{, j}}=A_{i j} \varphi_{, i} \\
S=-\frac{\partial W}{\partial \theta}=-a_{i j} e_{i j}-b_{i j} v_{i j}-c_{i j k} \chi_{i j k}+c \theta+m \varphi, \\
g=-\frac{\partial W}{\partial \varphi}=-\alpha_{i j} e_{i j}-\beta_{i j} v_{i j}-\delta_{i j k} \chi_{i j k}+m \theta-\xi \varphi,
\end{gathered}
$$

where we use the notation $S$ to designate the entropy (per unit mass).

We will consider that the above equations take place in the cylinder $\left[0, t_{0}\right) \times D$.

Denoting by $q$ the heat flux vector of components $q_{i}$, we can deduce a constitutive relation, similar to that from the classical theory, namely,

$$
q_{j}=K_{i j} \theta_{, i}
$$

where we denoted by $K_{i j}$ the tensor of the thermal conductivity and assume that $K_{i j}$ is a symmetric tensor.

In the absence of supply terms, the differential equations that govern the thermoelasticity of dipolar bodies with voids, obtained as in [5,6], are as follows.

The equations of motion:

$$
\begin{gathered}
\left(t_{i j}+\sigma_{i j}\right)_{, j}=\rho \ddot{u}_{i}, \\
\eta_{i j k, i}+\sigma_{j k}=I_{k r} \ddot{\phi}_{j r} ;
\end{gathered}
$$

The balance of the equilibrated forces:

$$
h_{i, i}+g=J \ddot{\varphi} ;
$$

The equation of energy:

$$
\rho T_{0} \dot{S}=q_{i, i}+\rho r
$$

In the preceding equations, we used some notions with following signification; $\rho$-the constant mass density, and $I_{i j}$-the tensor of microinertia, which is a symmetric tensor. 
$A_{i j m n}, B_{i j m n}, \ldots, a_{i j}$ are the coefficients that characterize the properties of the material with regards to the elasticity. Clearly, from (3), we can deduce the following symmetry relations,

$$
\begin{gathered}
A_{i j m n}=A_{j i m n}=A_{m n i j}, B_{i j m n}=B_{m n i j}, A_{i j}=A_{j i}, a_{i j}=a_{j i} \\
C_{i j k m n r}=C_{m n r i j k}, F_{i j k m n}=F_{i j k n m}, D_{i j m n}=D_{i j n m}, \alpha_{i j}=\alpha_{j i} .
\end{gathered}
$$

If we consider Equations (6)-(8), and then involve the kinematic equations (2) and the constitutive relations (4) and (5), from the equations of motion and the energy equations, we obtain the following system of equations,

$$
\begin{aligned}
& \rho \ddot{u}_{i}=\left[\left(C_{i j m n}+G_{i j m n}\right) e_{m n}+\left(G_{m n i j}+B_{i j m n}\right) v_{m n}+\right. \\
& \left.+\left(F_{m n r i j}+D_{i j m n r}\right) \chi_{m n r}+\left(a_{i j}+b_{i j}\right) \theta+\left(\alpha_{i j}+\beta_{i j}\right) \varphi\right]_{, j}, \\
& I_{k r} \ddot{\phi}_{j r}=\left[F_{i j k m n} e_{m n}+D_{m n i j k} v_{m n}+A_{i j k m n r} \chi_{m n r}+c_{i j k} \theta+\delta_{i j k} \varphi\right]_{, i}+ \\
& +G_{j k m n} e_{m n}+B_{j k m n} v_{m n}+D_{j k m n r} \chi_{m n r}+b_{j k} \theta+\beta_{j k} \varphi, \\
& c \dot{\theta}=-\left(K_{i j} \theta_{, i}\right)_{, j}+a_{i j} \dot{e}_{i j}+b_{i j} v_{i j}+c_{i j k} \dot{x}_{i j k}-m \dot{\varphi}, \\
& J \ddot{\varphi}=\left(A_{i j} \varphi_{, i}\right)_{, j}-\alpha_{i j} \dot{e}_{i j}-\beta_{i j} v_{i j}-\delta_{i j k} \dot{\chi}_{i j k}+m \theta-\xi \varphi,
\end{aligned}
$$

which take place for $(t, x) \in[0, \infty) \times D$.

We also suppose that the Equations (2) and (4) take place for $(t, x) \in[0, \infty) \times D$.

We now want to define a surface tractions vector of components $t_{i}$, a surface couple tensor of components $\eta_{j k}$, the flux of heat denoted by $q$, and the traction of the equilibrated stress vector $h$. For this we need the unit normal to the border $\partial D$, of components $n_{i}$, outward oriented. These have the following expressions,

$$
t_{i}=\left(t_{i j}+\sigma_{i j}\right) n_{j}, \eta_{j k}=\eta_{i j k} n_{i}, q=q_{i} n_{i}, h=h_{i} n_{i},
$$

and are defined at regular points of the border $\partial D$.

Having these tractions, we can now consider the following boundary data, in their nonhomogeneous form,

$$
\begin{aligned}
& u_{i}(t, x)=\tilde{u}_{i},(t, x) \in[0, \infty) \times \partial D_{u}, t_{i}(t, x)=\tilde{t}_{i},(t, x) \in[0, \infty) \times \in \partial D_{u}^{c}, \\
& \phi_{i j}(t, x)=\tilde{\phi}_{i j},(t, x) \in[0, \infty) \times \in \partial D_{\phi}, \eta_{j k}(t, x)=\tilde{\eta}_{j k},(t, x) \in[0, \infty) \times \in \partial D_{\phi}^{c}, \\
& \theta(t, x)=\tilde{\theta},(t, x) \in[0, \infty) \times \in \partial D_{\theta}, q(t, x)=\tilde{q},(t, x) \in[0, \infty) \times \in \partial D_{\theta}^{c}, \\
& \varphi(t, x)=\tilde{\varphi},(t, x) \in[0, \infty) \times \in \partial D_{\varphi}, h(t, x)=\tilde{h},(t, x) \in[0, \infty) \times \in \partial D_{\varphi^{\prime}}^{c},
\end{aligned}
$$

where the subsets $\partial D_{u}, \partial D_{\phi}, \partial D_{\theta}$, and $\partial D_{\varphi}$ and its complements $\partial D_{u}^{c}, \partial D_{\phi}^{c}, \partial D_{\theta}^{c}, \partial D_{\varphi}^{c}$ are surfaces from the boundary $\partial D$, having the following properties,

$$
\begin{gathered}
\partial \bar{D}_{u} \cup \partial D_{u}^{c}=\partial \bar{D}_{\phi} \cup \partial D_{\phi}^{c}=\partial \bar{D}_{\theta} \cup \partial D_{\theta}^{c}=\partial \bar{D}_{\varphi} \cup \partial D_{\varphi}^{c}=\partial D, \\
\partial D_{u} \cap \partial D_{u}^{c}=\partial D_{\phi} \cap \partial D_{\phi}^{c}=\partial D_{\theta} \cap \partial D_{\theta}^{c}=\partial D_{\varphi} \cap \partial D_{\varphi}^{c}=\varnothing .
\end{gathered}
$$

The mixed problem will be complete if we add the initial restrictions:

$$
\begin{array}{r}
u_{i}(x, 0)=u_{i}^{0}(x), \dot{u}_{i}(x, 0)=u_{i}^{1}(x), \theta(x, 0)=\theta^{0}(x), \\
\phi_{i j}(x, 0)=\phi_{i j}^{0}(x), \dot{\phi}_{i j}(x, 0)=\phi_{i j}^{1}(x), \varphi(x, 0)=\varphi^{0}(x),
\end{array}
$$

which take place on the closed domain $\bar{D}$. 
Here, $u_{i}^{0}(x), u_{i}^{1}(x), \phi_{i j}^{0}(x), \phi_{i j}^{1}(x), \theta^{0}(x)$, and $\varphi^{0}(x)$ are given functions and are assume be continuous. Furthermore, we must suppose that the above functions are adequacy with the restrictions (11) on the respective subsurfaces of $\partial D$.

By $\Psi$ we denote the internal energy and consider it as a quadratic form, as follows,

$$
\begin{aligned}
\Psi= & \frac{1}{2} A_{i j m n} e_{i j} e_{m n}+D_{i j m n} e_{i j} v_{m n}+F_{i j m n r} e_{i j} \chi_{m n r}+ \\
& +\frac{1}{2} B_{i j m n} v_{i j} v_{m n}+G_{i j m n r} v_{i j} \chi_{m n r}+\frac{1}{2} C_{i j k m n r} \chi_{i j k} \chi_{m n r} .
\end{aligned}
$$

Let us denote by $\mathcal{P}$ our initial boundary value problem, which includes the differential equations from (10), the initial restrictions from (12), and the data to the limit (11).

The results we want to expose will only be possible if certain restrictions are met, imposed on all the functions we will deal with.

First, we denote by $J_{m}(x)$ the smallest eigenvalue of the inertia tensor $I_{i j}(x)$, and we must suppose that $J_{m}$ is a continuous function. Also, the density $\rho$ and the constitutive coefficients are assumed be functions of class $C^{1}(D)$. Also, we must suppose that

(a) $\rho(x) \geq a_{1}, J_{m}(x) \geq a_{2}, c(x) \geq a_{3}$, where the real constants $a_{1}, a_{2}, a_{3}$ are positive;

(b) $K_{i j}$ is a positive definite tensor;

(c) the quadratic form $\Psi$ is positive definite.

As a consequence of the hypothesis (b), we can deduce the inequalities:

$$
K_{m} \theta_{, i} \theta_{, j} \leq K_{i j} \theta_{, i} \theta_{, j} \leq K_{M} \theta_{, i} \theta_{, j},
$$

where $K_{m}$ and $K_{M}$ are two positive constants.

If we take into account the hypothesis (c), then we can determine two positive numbers $M_{1}$ and $M_{2}$ to satisfy the double inequality that follows,

$$
\frac{M_{1}}{2}\left(e_{i j} e_{i j}+v_{i j} v_{i j}+\chi_{i j k} \chi_{i j k}\right) \leq \Psi \leq \frac{M_{2}}{2}\left(e_{i j} e_{i j}+v_{i j} v_{i j}+\chi_{i j k} \chi_{i j k}\right) .
$$

The above restrictions are commonly imposed in mechanics of solids, therefore they cannot be considered as very restrictive.

As such, our mixed problem $\mathcal{P}$ consists of the following restrictions and equations.

- $\quad$ the equations of motion $(10)_{1}$ and $(10)_{2}$, which take place in cylinder $D \times[0, \infty)$;

- the equation of energy:

$$
K_{i j}\left(\theta_{, j}\right)_{, i}=T_{0}\left[a_{i j} \dot{\mathrm{u}}_{i, j}+b_{i j}\left(\dot{\mathrm{u}}_{j, i}-\dot{\phi}_{i j}\right)+c_{i j k} \dot{\phi}_{i j, k}+c \dot{\theta}\right], \text { in }[0, \infty) \times D ;
$$

- $\quad$ the kinematic equations (2), which take place in cylinder $[0, \infty) \times D$;

- $\quad$ the constitutive conditions (4), which take place in cylinder $[0, \infty) \times D$;

- $\quad$ the initial restrictions (11), which take place in $\bar{D}$;

- the conditions to the limit:

$$
\begin{gathered}
\mathrm{u}_{i}(x, t)=0,(x, t) \in[0, \infty) \times \partial D_{u}, t_{i}=0,(x, t) \in[0, \infty) \times \partial D_{u \prime}^{c} \\
\phi_{i j}(x, t)=0,(x, t) \in[0, \infty) \times, \partial D_{\phi} m_{j k}=0,(x, t) \in[0, \infty) \times \partial D_{\phi^{\prime}}^{c} \\
\theta(x, t)=0,(x, t) \in[0, \infty) \times \partial D_{\theta}, q=0,(x, t) \in[0, \infty) \times \partial D_{\theta^{\prime}}^{c} \\
\varphi(x, t)=0,(x, t) \in[0, \infty) \times \partial D_{\varphi}, h=0,(x, t) \in[0, \infty) \times \partial D_{\varphi}^{c} .
\end{gathered}
$$




\section{Auxiliary Results}

At the beginning of this section, we will establish some identities of integral type, with regards to the solutions $\mathbf{u}=\left(u_{i}, \phi_{i j}, \theta, \varphi\right)$ of the above mixed problem $\mathcal{P}$. The important results of our study will be based on these identities.

Proposition 1. If $\mathbf{u}=\left(u_{i}, \phi_{i j}, \theta\right)$ is a solution of our above problem $\mathcal{P}^{\prime}$, then the following identity is satisfied

$$
\begin{array}{r}
\frac{1}{2} \int_{B}\left[\left(\rho \dot{u}_{i}(t) \dot{u}_{i}(t)+I_{j k} \dot{\phi}_{j m}(t) \dot{\phi}_{k m}(t)\right)+J \dot{\varphi}^{2}(t)+c \theta^{2}(t)\right] d V+ \\
+\int_{B}\left[\frac{1}{2} A_{i j m n} e_{i j}(t) e_{m n}(t)+D_{i j m n} e_{i j}(t) v_{m n}(t)+F_{i j m n r} e_{i j}(t) \chi_{m n r}(t)+\right. \\
\left.+\frac{1}{2} B_{i j m n} v_{i j}(t) v_{m n}(t)+G_{i j m n r} v_{i j}(t) \chi_{m n r}(t)+\frac{1}{2} C_{i j k m n r} \chi_{i j k}(t) \chi_{m n r}(t)\right] d V+ \\
+\int_{B}\left[A_{i j} \varphi_{, i} \varphi_{, j}+\alpha_{i j} e_{i j} \varphi+\beta_{i j} v_{i j} \varphi+\delta_{i j k} \chi_{i j k} \varphi+\xi \varphi^{2}\right) d V= \\
+\int_{0}^{t} \int_{D} \frac{1}{T_{0}} K_{i j} \theta_{, i}(s) \theta_{, j}(s) d V d s, \forall t \geq 0 .
\end{array}
$$

Proof. We multiply the equations of motion $(10)_{1}$ by $\dot{u}_{i}$ and consider the kinematic Equation (2), after simple calculations, the following identity is obtained,

$$
\begin{aligned}
\rho \dot{u}_{i} \dot{u}_{i}=\{[ & \left(A_{i j m n}+G_{i j m n}\right) e_{m n}+\left(G_{m n i j}+B_{i j m n}\right) v_{m n}+ \\
& \left.\left.+\left(F_{m n r i j}+D_{i j m n r}\right) \chi_{m n r}+\left(a_{i j}+b_{i j}\right) \theta+\left(\alpha_{i j}+\beta_{i j}\right) \varphi\right] \dot{u}_{i}\right\}_{, j}- \\
- & {\left[\left(A_{i j m n}+G_{i j m n}\right) e_{m n}+\left(G_{m n i j}+B_{i j m n}\right) v_{m n}+\right.} \\
& \left.\quad+\left(F_{m n r i j}+D_{i j m n r}\right) \chi_{m n r}+\left(a_{i j}+b_{i j}\right) \theta+\left(\alpha_{i j}+\beta_{i j}\right) \varphi\right] \dot{u}_{i, j} .
\end{aligned}
$$

If we multiply the equations of motion (10) 2 by $\dot{\phi}_{i j}$ and consider the kinematic Equation (2), after simple calculations, the following identity is obtained,

$$
\begin{aligned}
I_{j k} \dot{\phi}_{j m} \dot{\phi}_{k m}= & {\left[\left(F_{i j k m n} e_{m n}+D_{m n i j k} v_{m n}+C_{i j k m n r} \chi_{m n r}+c_{i j k} \theta+\delta_{i j k} \varphi\right) \dot{\phi}_{j k}\right]_{, i}-} \\
& -\left[F_{i j k m n} e_{m n}+D_{m n i j k} v_{m n}+C_{i j k m n r} \chi_{m n r}+c_{i j k} \theta+\delta_{i j k} \varphi\right] \dot{\phi}_{j k, i}+ \\
+ & {\left[G_{j k m n} e_{m n}+B_{j k m n} v_{m n}+D_{j k m n r} \chi_{m n r}+b_{j k} \theta+\beta_{j k} \varphi\right] \phi_{j k} . }
\end{aligned}
$$

Now we multiply the equations of motion $(10)_{3}$ by $\theta$. After simple calculations the following identity is obtained,

$$
\begin{aligned}
c \theta \dot{\theta}= & -\left(K_{i j} \theta_{i} \theta\right)_{, j}+K_{i j} \theta_{, i} \theta_{, j}+ \\
& +a_{i j} \dot{e}_{i j} \theta+b_{i j} \dot{v}_{i j} \theta+c_{i j k} \dot{\chi}_{i j k} \theta-m \theta \dot{\varphi} .
\end{aligned}
$$

Finally, we multiply the equations of motion $(10)_{4}$ by $\varphi$, so that after simple calculations, the following identity is obtained,

$$
\begin{aligned}
J \dot{\varphi} \ddot{\varphi}= & \left(A_{i j} \varphi_{, i} \dot{\varphi}\right)_{, j}-A_{i j} \varphi_{, i} \dot{\varphi}_{, j}- \\
& -\alpha_{i j} e_{i j} \dot{\varphi}-\beta_{i j} v_{i j} \dot{\varphi}-\delta_{i j k} \chi_{i j k} \dot{\varphi}-\xi \varphi \dot{\varphi}+m \theta \dot{\varphi} .
\end{aligned}
$$


Now we sum up, term with term, the equalities (19)-(22) and take into account the geometric relations (5) so that we reach the equality

$$
\begin{aligned}
& \rho \dot{u}_{i} \ddot{u}_{i}+I_{j k} \dot{\phi}_{j m} \ddot{\phi}_{k m}+c \theta \dot{\theta}+J \dot{\varphi} \ddot{\varphi}= \\
&=\left\{\left[\left(A_{i j m n}+G_{i j m n}\right) e_{m n}+\left(G_{m n i j}+B_{i j m n}\right) v_{m n}+\right.\right. \\
&\left.\left.+\left(F_{m n r i j}+D_{i j m n r}\right) \chi_{m n r}+\left(a_{i j}+b_{i j}\right) \theta+\left(\alpha_{i j}+\beta_{i j}\right) \varphi\right] \dot{u}_{i}\right\}_{, j}+ \\
&+ {\left[\left(F_{i j k m n} e_{m n}+D_{m n i j k} v_{m n}+C_{i j k m n r} \chi_{m n r}+c_{i j k} \theta+\delta_{i j k} \varphi\right) \dot{\phi}_{j k}\right]_{, i}-} \\
&- {\left[\left(A_{i j m n}+G_{i j m n}\right) e_{m n}+\left(G_{m n i j}+B_{i j m n}\right) v_{m n}+\right.} \\
&\left.+\left(F_{m n r i j}+D_{i j m n r}\right) \chi_{m n r}+\left(a_{i j}+b_{i j}\right) \theta+\left(\alpha_{i j}+\beta_{i j}\right) \varphi\right] \dot{u}_{i, j}- \\
&-\left[F_{i j k m n} e_{m n}+D_{m n i j k} v_{m n}+C_{i j k m n r} \chi_{m n r}+c_{i j k} \theta+\delta_{i j k} \varphi\right] \dot{\phi}_{j k, i}+ \\
& \quad+\left[G_{j k m n} e_{m n}+B_{j k m n} v_{m n}+D_{j k m n r} \chi_{m n r}+b_{j k} \theta+\beta_{j k} \varphi\right] \phi_{j k}- \\
& \quad-\left(K_{i j} \theta_{, i} \theta\right)_{, j}+K_{i j} \theta_{, i} \theta_{, j}+a_{i j} \dot{e}_{i j} \theta+b_{i j} \dot{v}_{i j} \theta+c_{i j k} \dot{\chi}_{i j k} \theta+ \\
&+\left(A_{i j} \varphi_{, i} \dot{\varphi}\right)_{, j}-A_{i j} \varphi_{, i} \dot{\varphi}_{, j}+\alpha_{i j} e_{i j} \dot{\varphi}+\beta_{i j} v_{i j} \dot{\varphi}+\delta_{i j k} \chi_{i j k} \dot{\varphi}-\xi \varphi \dot{\varphi},
\end{aligned}
$$

which can be restated in the following form

$$
\begin{gathered}
\rho \dot{u}_{i} \ddot{u}_{i}+I_{j k} \dot{\phi}_{j m} \ddot{\phi}_{k m}+c \theta \dot{\theta}+J \dot{\varphi} \ddot{\varphi}+ \\
+A_{i j m n} e_{m n} \dot{e}_{i j}+G_{m n i j}\left(v_{m n} \dot{e}_{i j}+\dot{v}_{m n} e_{i j}\right)+F_{m n r i j}\left(\chi_{m n r} \dot{e}_{i j}+\dot{\chi}_{m n r} e_{i j}\right) \\
+B_{i j m n} v_{m n} \dot{v}_{i j}+D_{m n i j k} v_{m n}\left(v_{m n} \dot{\chi}_{i j k}+\dot{v}_{m n} \chi_{i j k}\right)+C_{i j k m n r} \chi_{m n r} \dot{\chi}_{i j k}+ \\
+\alpha_{i j}\left(e_{i j} \dot{\varphi}+\dot{e}_{i j} \varphi\right)+\beta_{i j}\left(v_{i j} \dot{\varphi}+\dot{v}_{i j} \varphi\right)+\delta_{i j k}\left(\chi_{i j k} \dot{\varphi}+\dot{\chi}_{i j k} \varphi\right)+ \\
+A_{i j} \varphi_{, i} \dot{\varphi}_{, j}+\xi \varphi \dot{\varphi}= \\
=\left\{\left[\left(A_{i j m n}+G_{i j m n}\right) e_{m n}+\left(G_{m n i j}+B_{i j m n}\right) v_{m n}+\right.\right. \\
\left.\left.+\left(F_{m n r i j}+D_{i j m n r}\right) \chi_{m n r}+\left(a_{i j}+b_{i j}\right) \theta+\left(\alpha_{i j}+\beta_{i j}\right) \varphi\right] \dot{u}_{i}\right\}_{, j}+ \\
+\left[\left(F_{i j k m n} e_{m n}+D_{m n i j k} v_{m n}+C_{i j k m n r} \chi_{m n r}+c_{i j k} \theta+\delta_{i j k} \varphi\right) \dot{\phi}_{j k}\right]_{, i}+ \\
+\left(A_{i j} \varphi_{, i} \dot{\varphi}\right)_{, j}-\left(K_{i j} \theta_{, i} \theta\right)_{, j}+K_{i j} \theta_{, i} \theta_{, j} .
\end{gathered}
$$

We can now integrate this identity over domain $[0, t] \times D$. Taking into account the divergence theorem and considering that we have null boundary data, we obtain the identity (18), as such the proof of Proposition 1 is finished.

A new energy relation is obtained in next proposition. We consider the case of null initial data.

Proposition 2. Let $\left(u_{i}, \phi_{i j}, \theta, \varphi\right)$ be a solution of the mixed problem, $\mathcal{P}$, corresponding to zero initial conditions. Then, the following equality is satisfied:

$$
\begin{aligned}
\frac{1}{2} \int_{B} & \left(\rho \dot{u}_{i} \dot{u}_{i}+I_{j k} \dot{\phi}_{j m} \dot{\phi}_{k m}+J \dot{\varphi}^{2}-c \theta^{2}\right) d V+ \\
& +\int_{B}\left(\frac{1}{2} A_{i j m n} e_{i j} e_{m n}+D_{i j m n} e_{i j} v_{m n}+F_{i j m n r} e_{i j} \chi_{m n r}+\right. \\
& \left.+\frac{1}{2} B_{i j m n} v_{i j} v_{m n}+G_{i j m n r} v_{i j} \chi_{m n r}+\frac{1}{2} C_{i j k m n r} \chi_{i j k} \chi_{m n r}\right) d V+ \\
& +\int_{B}\left(\frac{1}{2} A_{i j} \varphi_{, i} \varphi_{, j}+\alpha_{i j} e_{i j} \varphi+\beta_{i j} v_{i j} \varphi+\delta_{i j k} \chi_{i j k} \varphi+\frac{1}{2} \xi \varphi^{2}\right) d V= \\
= & -\int_{0}^{t} \int_{D}\left(K_{i j} \theta_{, i} \theta_{, j}-a_{i j} \dot{e}_{i j} \theta-b_{i j} \dot{v}_{i j} \theta-c_{i j k} \dot{\chi}_{i j k} \theta-m \theta \dot{\varphi}\right) d V d \tau,
\end{aligned}
$$


for any $t \in[0, \infty)$.

Proof. If we multiply the equation of motion $(10)_{1}$ by $\dot{u}_{i}$, then Equation (19) is obtained. If we multiply the equation of motion (10) $)_{2}$ by $\dot{\phi}_{i j}$, then Equation (20) is obtained. If we multiply the equation of motion (10) $)_{4}$ by $\dot{\varphi}$, then Equation (22) is obtained. Now, we multiply the equation of motion $(10)_{3}$ by $-\theta$ so that the following identity is obtained,

$$
\begin{aligned}
-c \theta \dot{\theta} & =\left(K_{i j} \theta_{, i} \theta\right)_{, j}-K_{i j} \theta_{, i} \theta_{, j}- \\
& -a_{i j} \dot{e}_{i j} \theta-b_{i j} \dot{v}_{i j} \theta-c_{i j k} \dot{\chi}_{i j k} \theta+m \theta \dot{\varphi} .
\end{aligned}
$$

From Equations (19), (20), (22), and (26) we deduce

$$
\begin{gathered}
\rho \dot{u}_{i} \ddot{u}_{i}+I_{j k} \dot{\phi}_{j m} \ddot{\phi}_{k m}-c \theta \dot{\theta}+J \dot{\varphi} \ddot{\varphi}+ \\
+A_{i j m n} e_{m n} \dot{e}_{i j}+G_{m n i j}\left(v_{m n} \dot{e}_{i j}+\dot{v}_{m n} e_{i j}\right)+F_{m n r i j}\left(\chi_{m n r} \dot{e}_{i j}+\dot{\chi}_{m n r} e_{i j}\right) \\
+B_{i j m n} v_{m n} \dot{v}_{i j}+D_{m n i j k} v_{m n}\left(v_{m n} \dot{\chi}_{i j k}+\dot{v}_{m n} \chi_{i j k}\right)+C_{i j k m n r} \chi_{m n r} \dot{\chi}_{i j k}+ \\
+\alpha_{i j}\left(e_{i j} \dot{\varphi}+\dot{e}_{i j} \varphi\right)+\beta_{i j}\left(v_{i j} \dot{\varphi}+\dot{v}_{i j} \varphi\right)+\delta_{i j k}\left(\chi_{i j k} \dot{\varphi}+\dot{\chi}_{i j k} \varphi\right)+ \\
+A_{i j} \varphi_{, i} \dot{\varphi}_{, j}+\xi \varphi \dot{\varphi}= \\
=\left\{\left[\left(A_{i j m n}+G_{i j m n}\right) e_{m n}+\left(G_{m n i j}+B_{i j m n}\right) v_{m n}+\right.\right. \\
\left.\left.+\left(F_{m n r i j}+D_{i j m n r}\right) \chi_{m n r}+\left(a_{i j}+b_{i j}\right) \theta+\left(\alpha_{i j}+\beta_{i j}\right) \varphi\right] \dot{u}_{i}\right\}_{, j}+ \\
+\left[\left(F_{i j k m n} e_{m n}+D_{m n i j k} v_{m n}+C_{i j k m n r} \chi_{m n r}+c_{i j k} \theta+\delta_{i j k} \varphi\right) \dot{\phi}_{j k}\right]_{, i}+ \\
+\left(A_{i j} \varphi_{, i} \dot{\varphi}\right)_{, j}+\left(K_{i j} \theta_{, i} \theta\right)_{, j}-K_{i j} \theta_{, i} \theta_{, j}+ \\
+a_{i j} \dot{e}_{i j} \theta+b_{i j} \dot{v}_{i j} \theta+c_{i j k} \dot{\chi}_{i j k} \theta+m \theta \dot{\varphi} .
\end{gathered}
$$

Finally, we integrate the last identity over domain $[0, t] \times D$, so that considering the divergence theorem and taking into account that we have null initial data, we obtain the identity (25), as such the proof of Proposition 2 is finished.

In the following proposition, we deduce another energy relation, obtained by a method of the Lagrange identity type.

Proposition 3. Let $\left(u_{i}, \phi_{i j}, \theta, \varphi\right)$ be a solution of the mixed problem $\mathcal{P}$, corresponding to zero initial conditions and null boundary data. Then, the following equality is satisfied,

$$
\begin{aligned}
\int_{B}( & \left.\rho \dot{u}_{i} \dot{u}_{i}+I_{j k} \dot{\phi}_{j m} \dot{\phi}_{k m}+J \dot{\varphi}^{2}-c \theta^{2}\right) d V= \\
= & \int_{B}\left(A_{i j m n} e_{i j} e_{m n}+2 D_{i j m n} e_{i j} v_{m n}+2 F_{i j m n r} e_{i j} \chi_{m n r}+\right. \\
\quad & \left.\quad B_{i j m n} v_{i j} v_{m n}+2 G_{i j m n r} v_{i j} \chi_{m n r}+C_{i j k m n r} \chi_{i j k} \chi_{m n r}\right) d V+ \\
\quad & \quad \int_{B}\left(A_{i j} \varphi_{, i} \varphi_{, j}+2 \alpha_{i j} e_{i j} \varphi+2 \beta_{i j} v_{i j} \varphi+2 \delta_{i j k} \chi_{i j k} \varphi+\xi \varphi^{2}\right) d V,
\end{aligned}
$$

for any $t \in[0, \infty)$. 
Proof. Let us consider the Equation $(10)_{1}$ and fix an arbitrary $t \in(0, \infty)$. By simple calculations, we reach the identity

$$
\begin{aligned}
& \frac{\partial}{\partial \tau}\left[\rho \dot{u}_{i}(\tau) \dot{u}_{i}(2 t-\tau)\right]=\rho \ddot{u}_{i}(\tau) \dot{u}_{i}(2 t-\tau)-\rho \dot{u}_{i}(\tau) \ddot{u}_{i}(2 t-\tau)= \\
& =\left\{\left[\left(A_{i j m n}+G_{i j m n}\right) e_{m n}(\tau)+\left(G_{m n i j}+B_{i j m n}\right) v_{m n}(\tau)+\right.\right. \\
& \left.\left.+\left(F_{m n r i j}+D_{i j m n r}\right) \chi_{m n r}(\tau)+\left(a_{i j}+b_{i j}\right) \theta(\tau)+\left(\alpha_{i j}+\beta_{i j}\right) \varphi(\tau)\right] \dot{u}_{i}(2 t-\tau)\right\}_{, j}- \\
& \quad-\left[\left(A_{i j m n}+G_{i j m n}\right) e_{m n}(\tau)+\left(G_{m n i j}+B_{i j m n}\right) v_{m n}(\tau)+\right. \\
& \left.\quad+\left(F_{m n r i j}+D_{i j m n r}\right) \chi_{m n r}(\tau)+\left(a_{i j}+b_{i j}\right) \theta(\tau)+\left(\alpha_{i j}+\beta_{i j}\right) \varphi(\tau)\right] \dot{u}_{i, j}(2 t-\tau)- \\
& -\left\{\left[\left(A_{i j m n}+G_{i j m n}\right) e_{m n}(2 t-\tau)+\left(G_{m n i j}+B_{i j m n}\right) v_{m n}(2 t-\tau)+\right.\right. \\
& \left.\left.+\left(F_{m n r i j}+D_{i j m n r}\right) \chi_{m n r}(2 t-\tau)+\left(a_{i j}+b_{i j}\right) \theta(2 t-\tau)+\left(\alpha_{i j}+\beta_{i j}\right) \varphi(2 t-\tau)\right] \dot{u}_{i}(\tau)\right\}_{, j}+ \\
& \quad+\left[\left(A_{i j m n}+G_{i j m n}\right) e_{m n}(2 t-\tau)+\left(G_{m n i j}+B_{i j m n}\right) v_{m n}(2 t-\tau)+\right. \\
& \left.\quad+\left(F_{m n r i j}+D_{i j m n r}\right) \chi_{m n r}(2 t-\tau)+\left(a_{i j}+b_{i j}\right) \theta(2 t-\tau)+\left(\alpha_{i j}+\beta_{i j}\right) \varphi(2 t-\tau)\right] \dot{u}_{i, j}(\tau) .
\end{aligned}
$$

Now we take into account the Equation $(10)_{2}$, for an arbitrary fixed $t \in(0, \infty)$. After some calculations, we reach the identity

$$
\begin{aligned}
& \frac{\partial}{\partial \tau}[\left.I_{j k} \dot{\phi}_{j m}(\tau) \dot{\phi}_{k m}(2 t-\tau)\right]=I_{j k} \ddot{\phi}_{j m}(\tau) \dot{\phi}_{k m}(2 t-\tau)-I_{j k} \dot{\phi}_{j m}(\tau) \ddot{\phi}_{k m}(2 t-\tau)= \\
&=\left\{\left[F_{i j k m n} e_{m n}(\tau)+D_{m n i j k} v_{m n}(\tau)+C_{i j k m n r} \chi_{m n r}(\tau)+c_{i j k} \theta(\tau)+\delta_{i j k} \varphi(\tau)\right] \dot{\phi}_{j k}(\alpha)\right\}_{, i}- \\
&-\left[F_{i j k m n} e_{m n}(\tau)+D_{m n i j k} v_{m n}(\tau)+C_{i j k m n r} \chi_{m n r}(\tau)+c_{i j k} \theta(\tau)+\delta_{i j k} \varphi(\tau)\right] \dot{\phi}_{j k, i}(\alpha)+ \\
&+\left[G_{j k m n} e_{m n}(\tau)+B_{j k m n} v_{m n}(\tau)+D_{j k m n r} \chi_{m n r}(\tau)+b_{j k} \theta(\tau)+\beta_{j k} \varphi(\tau)\right] \phi_{j k}(\alpha)- \\
&-\left\{\left[F_{i j k m n} e_{m n}(\alpha)+D_{m n i j k} v_{m n}(\alpha)+C_{i j k m n r} \chi_{m n r}(\alpha)+c_{i j k} \theta(\alpha)+\delta_{i j k} \varphi(\alpha)\right] \dot{\phi}_{j k}(\tau)\right\}_{, i}+ \\
&+ \\
&+\left[F_{i j k m n} e_{m n}(\alpha)+D_{m n i j k} v_{m n}(\alpha)+C_{i j k m n r} \chi_{m n r}(\alpha)+c_{i j k} \theta(\alpha)+\delta_{i j k} \varphi(\alpha)\right] \dot{\phi}_{j k, i}(\tau) \\
& \quad-\left[G_{j k m n} e_{m n}(\alpha)+B_{j k m n} v_{m n}(\alpha)+D_{j k m n r} \chi_{m n r}(\alpha)+b_{j k} \theta(\alpha)+\beta_{j k} \varphi(\alpha)\right] \phi_{j k}(\tau),
\end{aligned}
$$

where, to simplify the writing, we used the notation $2 t-\tau=\alpha$.

Now, similarly, for an arbitrarily fixed $t \in(0, \infty)$ we consider the Equation $(10)_{3}$ to deduce

$$
\begin{aligned}
& \frac{\partial}{\partial \tau}[c \theta(\tau) \theta(2 t-\tau)]=c \dot{\theta}(\tau) \theta(2 t-\tau)-c \theta(\tau) \dot{\theta}(2 t-\tau)= \\
& =-\left(K_{i j} \theta_{, i}(\tau) \theta(2 t-\tau)\right)_{, j}+\left[a_{i j} \dot{e}_{i j}(\tau)+b_{i j} \dot{v}_{i j}(\tau)+c_{i j k} \dot{\chi}_{i j k}(\tau)-m \dot{\varphi}(\tau)\right] \theta(2 t-\tau)+ \\
& \quad+\left(K_{i j} \theta_{, i}(2 t-\tau) \theta(\tau)\right)_{, j}-\left[a_{i j} \dot{e}_{i j}(2 t-\tau)+b_{i j} \dot{v}_{i j}(2 t-\tau)+c_{i j k} \dot{\chi}_{i j k}(2 t-\tau)-m \dot{\varphi}(2 t-\tau)\right] \theta(\tau) .
\end{aligned}
$$

Finally, for an arbitrarily fixed $t \in(0, \infty)$ we will use the Equation $(10)_{4}$ to obtain

$$
\begin{aligned}
& \frac{\partial}{\partial \tau}[J \dot{\varphi}(\tau) \dot{\varphi}(2 t-\tau)]=J \ddot{\varphi}(\tau) \dot{\varphi}(2 t-\tau)-J \dot{\varphi}(\tau) \ddot{\varphi}(2 t-\tau)= \\
& =\left(A_{i j} \varphi_{, i}(\tau) \dot{\varphi}(2 t-\tau)\right)_{, j}-A_{i j} \varphi_{, i}(\tau) \dot{\varphi}_{, j}(2 t-\tau)-\alpha_{i j} e_{i j}(\tau) \dot{\varphi}(2 t-\tau)- \\
& -\beta_{i j} v_{i j}(\tau) \dot{\varphi}(2 t-\tau)-\delta_{i j k} \chi_{i j k}(\tau) \dot{\varphi}(2 t-\tau)-\xi \varphi(\tau) \dot{\varphi}(2 t-\tau)+m \theta(\tau) \dot{\varphi}(2 t-\tau)- \\
& -\left(A_{i j} \varphi_{, i}(2 t-\tau) \dot{\varphi}(\tau)\right)_{, j}+A_{i j} \varphi_{, i}(2 t-\tau) \dot{\varphi}_{, j}(\tau)+\alpha_{i j} e_{i j}(2 t-\tau) \dot{\varphi}(\tau)+ \\
& +\beta_{i j} v_{i j}(2 t-\tau) \dot{\varphi}(\tau)+\delta_{i j k} \chi_{i j k}(2 t-\tau) \dot{\varphi}(\tau)+\xi \varphi(2 t-\tau) \dot{\varphi}(\tau)-m \theta(2 t-\tau) \dot{\varphi}(\tau) .
\end{aligned}
$$


Now we use the geometric Equation (2) so that from Equations (29)-(32) we are led to the identity

$$
\begin{array}{r}
\frac{\partial}{\partial \tau}\left[\rho \dot{u}_{i}(\tau) \dot{u}_{i}(2 t-\tau)+I_{j k} \dot{\phi}_{j m}(\tau) \dot{\phi}_{k m}(2 t-\tau)-c \theta(\tau) \theta(2 t-\tau)+J \dot{\varphi}(\tau) \dot{\varphi}(2 t-\tau)\right]= \\
+\left\{\left[\left(A_{i j m n}+G_{i j m n}\right) e_{m n}(\tau)+\left(G_{m n i j}+B_{i j m n}\right) v_{m n}(\tau)+\right.\right. \\
\left.\left.+\left(F_{m n r i j}+D_{i j m n r}\right) \chi_{m n r}(\tau)+\left(a_{i j}+b_{i j}\right) \theta(\tau)+\left(\alpha_{i j}+\beta_{i j}\right) \varphi(\tau)\right] \dot{u}_{i}(2 t-\tau)\right\}_{, j}- \\
-\left\{\left[\left(A_{i j m n}+G_{i j m n}\right) e_{m n}(2 t-\tau)+\left(G_{m n i j}+B_{i j m n}\right) v_{m n}(2 t-\tau)+\right.\right. \\
\left.\left.+\left(F_{m n r i j}+D_{i j m n r}\right) \chi_{m n r}(2 t-\tau)+\left(a_{i j}+b_{i j}\right) \theta(2 t-\tau)+\left(\alpha_{i j}+\beta_{i j}\right) \varphi(2 t-\tau)\right] \dot{u}_{i}(\tau)\right\}_{, j}+ \\
+\left\{\left[F_{i j k m n} e_{m n}(\tau)+D_{m n i j k} v_{m n}(\tau)+C_{i j k m n r} \chi_{m n r}(\tau)+c_{i j k} \theta(\tau)+\delta_{i j k} \varphi(\tau)\right] \dot{\phi}_{j k}(2 t-\tau)\right\}_{, i}- \\
-\left\{\left[F_{i j k m n} e_{m n}+D_{m n i j k} v_{m n}+C_{i j k m n r} \chi_{m n r}+c_{i j k} \theta+\delta_{i j k} \varphi\right](2 t-\tau) \dot{\phi}_{j k}(\tau)\right\}_{, i}+ \\
+\left(K_{i j} \theta_{, j}(\tau) \theta(2 t-\tau)\right)_{, i}-\left(K_{i j} \theta_{, i}(2 t-\tau) \theta(\tau)\right)_{, j}+\left(A_{i j} \varphi, i(\tau) \dot{\varphi}(2 t-\tau)\right)_{, j}-\left(A_{i j} \varphi, i(2 t-\tau) \dot{\varphi}(\tau)\right)_{, j} \\
+\frac{\partial}{\partial \tau}\left\{A_{i j m n} e_{i j}(\tau) e_{m n}(2 t-\tau)+D_{i j m n}\left[e_{i j}(\tau) v_{m n}(2 t-\tau)+e_{i j}(2 t-\tau) v_{m n}(\tau)\right]+\right. \\
+F_{i j k m n}\left[e_{m n}(\tau) \chi_{i j k}(2 t-\tau)+e_{m n}(2 t-\tau) \chi_{i j k}(\tau)\right]+B_{i j m n} v_{i j}(\tau) v_{m n}(2 t-\tau)+ \\
\left.+G_{i j k m n}\left[v_{m n}(\tau) \chi_{i j k}(2 t-\tau)+v_{m n}(2 t-\tau) \chi_{i j k}(\tau)\right]+C_{i j k m n r} \chi_{i j k}(\tau) \chi_{m n r}(2 t-\tau)\right\}+ \\
+\frac{\partial}{\partial \tau}\left\{\alpha_{i j}\left[e_{i j}(2 t-\tau) \dot{\varphi}(\tau)+e_{i j}(\tau) \dot{\varphi}(2 t-\tau)\right]+\beta_{i j}\left[v_{i j}(2 t-\tau) \dot{\varphi}(\tau)+v_{i j}(\tau) \dot{\varphi}(2 t-\tau)\right]\right\}+ \\
+\frac{\partial}{\partial \tau}\left\{\delta_{i j k}\left[\chi_{i j k}(2 t-\tau) \dot{\varphi}(\tau)+\chi_{i j k}(\tau) \dot{\varphi}(2 t-\tau)\right]+\xi \varphi(2 t-\tau) \dot{\varphi}(\tau)\right\} .
\end{array}
$$

This last equality is integrated over $[0, t] \times D$. Applying the divergence theorem and taking into account the initial conditions (12) and the boundary data (17), we arrive at the desired identity (28). As such, the proof of proposition is finished.

\section{On Localization in Time of Solutions}

The following theorem is essential to obtain the main result of our study.

Theorem 1. Suppose the main hypotheses (a)-(c) are satisfied. If $\left(u_{i}, \phi_{i j}, \theta, \varphi\right)$ is a solution of the mixed problem $\mathcal{P}$, which corresponds to zero initial conditions (12) and null boundary data (17), then this solution can only be null, that is,

$$
u_{i}(t, x)=0, \phi_{i j}(t, x)=0, \theta(t, x)=0, \varphi(t, x)=0, \forall(t, x) \in[0, \infty) \times D .
$$

Proof. By combining the results from Equations (25) and (28), we obtain

$$
\begin{aligned}
\int_{B} & \left(A_{i j m n} e_{i j} e_{m n}+2 D_{i j m n} e_{i j} v_{m n}+2 F_{i j m n r} e_{i j} \chi_{m n r}+\right. \\
& \left.+B_{i j m n} v_{i j} v_{m n}+2 G_{i j m n r} v_{i j} \chi_{m n r}+C_{i j k m n r} \chi_{i j k} \chi_{m n r}\right) d V+ \\
& +\int_{B}\left(A_{i j} \varphi_{, i} \varphi_{, j}+2 \alpha_{i j} e_{i j} \varphi+2 \beta_{i j} v_{i j} \varphi+2 \delta_{i j k} \chi_{i j k} \varphi+\xi \varphi^{2}\right) d V= \\
= & -\int_{0}^{t} \int_{D}\left(K_{i j} \theta_{, i} \theta_{, j}-a_{i j} \dot{e}_{i j} \theta-b_{i j} \dot{v}_{i j} \theta-c_{i j k} \dot{\chi}_{i j k} \theta-m \theta \dot{\varphi}\right) d V d \tau,
\end{aligned}
$$

for any $t \in[0, \infty)$. 
Inspired by the identities (18) and (35), we introduce the functions $G, E$ and $F$, defined by

$$
\begin{aligned}
& E(t)=\frac{1}{2} \int_{B}[\left.\left(\rho \dot{u}_{i} \dot{u}_{i}+I_{j k} \dot{\phi}_{j m} \dot{\phi}_{k m}\right)+J \dot{\varphi}^{2}+c \theta^{2}\right] d V+ \\
&+ \int_{B}\left[\frac{1}{2} A_{i j m n} e_{i j} e_{m n}+D_{i j m n} e_{i j} v_{m n}+F_{i j m n r} e_{i j} \chi_{m n r}+\right. \\
&\left.+\frac{1}{2} B_{i j m n} v_{i j} v_{m n}+G_{i j m n r} v_{i j} \chi_{m n r}+\frac{1}{2} C_{i j k m n r} \chi_{i j k} \chi_{m n r}\right] d V+ \\
&+\int_{B}\left[\frac{1}{2} A_{i j} \varphi_{, i} \varphi_{, j}+\alpha_{i j} e_{i j} \varphi+\beta_{i j} v_{i j} \varphi+\delta_{i j k} \chi_{i j k} \varphi+\frac{1}{2} \xi \varphi^{2}\right] d V \\
& G(t)=\int_{B}\left[\frac{1}{2} A_{i j m n} e_{i j} e_{m n}+D_{i j m n} e_{i j} v_{m n}+F_{i j m n r} e_{i j} \chi_{m n r}+\right. \\
&+\left.\frac{1}{2} B_{i j m n} v_{i j} v_{m n}+G_{i j m n r} v_{i j} \chi_{m n r}+\frac{1}{2} C_{i j k m n r} \chi_{i j k} \chi_{m n r}\right] d V+ \\
&+\int_{B}\left[\frac{1}{2} A_{i j} \varphi_{, i} \varphi_{, j}+\alpha_{i j} e_{i j} \varphi+\beta_{i j} v_{i j} \varphi+\delta_{i j k} \chi_{i j k} \varphi+\frac{1}{2} \xi \varphi^{2}\right] d V, \\
& F(t)=G(t)+\alpha E(t),
\end{aligned}
$$

for any $t \in[0, \infty)$.

In (36) $3 \alpha$ is a positive constant, conveniently of small chosen.

It is easy to see that $F(t)$ can be rewritten in the form

$$
\begin{gathered}
F(t)=\frac{1}{2} \int_{B}\left[\alpha\left(\rho \dot{u}_{i} \dot{u}_{i}+I_{j k} \dot{\phi}_{j m} \dot{\phi}_{k m}+J \dot{\varphi}^{2}+c \theta^{2}\right)+\right. \\
+(2+\alpha)\left(A_{i j m n} e_{i j} e_{m n}+2 D_{i j m n} e_{i j} v_{m n}+2 F_{i j m n r} e_{i j} \chi_{m n r}+\right. \\
\quad+B_{i j m n} v_{i j} v_{m n}+2 G_{i j m n r} v_{i j} \chi_{m n r}+C_{i j k m n r} \chi_{i j k} \chi_{m n r}+ \\
\left.\left.\quad+A_{i j} \varphi_{, i} \varphi_{, j}+2 \alpha_{i j} e_{i j} \varphi+2 \beta_{i j} v_{i j} \varphi+2 \delta_{i j k} \chi_{i j k} \varphi+\xi \varphi^{2}\right)\right] d V .
\end{gathered}
$$

Based on Equations (35) and (37), the function $F$ receives the following from

$$
\begin{aligned}
F(t)= & (\alpha-1) \int_{0}^{t} \int_{D} K_{i j} \theta_{, i} \theta_{, j} d V d \tau- \\
& -2 \int_{0}^{t} \int_{D}\left(a_{i j} \dot{e}_{i j} \theta+b_{i j} \dot{v}_{i j} \theta+c_{i j k} \dot{\chi}_{i j k} \theta\right) d V d \tau-2 \int_{0}^{t} \int_{D} m \theta \dot{\varphi} d V d \tau,
\end{aligned}
$$

so we can immediately deduce that

$$
\begin{aligned}
\frac{d F(t)}{d t}=(\alpha-1) & \int_{D} K_{i j} \theta_{, i} \theta_{, j} d V- \\
& -2 \int_{D}\left(a_{i j} \dot{e}_{i j} \theta+b_{i j} \dot{v}_{i j} \theta+c_{i j k} \dot{\chi}_{i j k} \theta\right) d V-2 \int_{D} m \theta \dot{\varphi} d V .
\end{aligned}
$$

Now, we can choose a sufficiently small positive constant $\beta$ and a positive constant $M_{1}$, computed in terms of $\beta$ and constitutive coefficients, so that

$$
\begin{array}{r}
\int_{D}\left(a_{i j} \dot{e}_{i j} \theta+b_{i j} \dot{v}_{i j} \theta+c_{i j k} \dot{\chi}_{i j k} \theta\right) d V \leq \beta \int_{D} K_{i j} \theta_{, i} \theta_{j} d V+ \\
+M_{1} \int_{D}\left(\rho \dot{u}_{i} \dot{u}_{i}+I_{j k} \dot{\phi}_{j m} \dot{\phi}_{k m}+J \dot{\varphi}^{2}+c \theta^{2}\right) d V
\end{array}
$$


On the other hand, can be determined the positive constant $M_{2}$ so that the following inequality takes place,

$$
\int_{D} m \theta \dot{\varphi} d V \leq M_{2} \int_{B}\left(J \dot{\varphi}^{2}+c \theta^{2}\right) d V .
$$

If we choose $\beta \leq 1-\alpha$ and take into account the inequalities (39)-(41), we can find a positive constant $M_{3}$ so that

$$
\frac{d F(t)}{d t} \leq M_{3} \int_{D}\left(\rho \dot{u}_{i} \dot{u}_{i}+I_{j k} \dot{\phi}_{j m} \dot{\phi}_{k m}+J \dot{\varphi}^{2}+c \theta^{2}\right) d V .
$$

Now, considering the inequalities (40)-(42) we can find a positive constant $c$ so that for every $t \geq 0$, inequality occurs

$$
\frac{d F(t)}{d t} \leq c F(t)
$$

from where by direct integration, we are led to the inequality

$$
F(t) \leq F(0) e^{c t}, \forall t \geq 0,
$$

so that we can deduce that $F(t)=0$, for any $t \geq 0$, taking into account the null initial data and the expression of $F(t)$ from (36).

Also, from (36), we deduce $\dot{u}_{i}(x, t)=0, \dot{\phi}_{i j}(x, t)=0, \dot{\theta}(x, t)=0, \dot{\varphi}(x, t)=0$, for any $t \geq 0, x \in D$.

Now the conclusion of Theorem 1 is easy to obtain, again considering that the initial data is null.

Finally, we want to formulate and prove the main theorem of our work. We will show that it is impossible to localizate in a finite time any solutions of our mixed problem $\mathcal{P}$ consisting of Equations (2), (4), and (6)-(8), the null initial conditions (12) and homogeneous boundary conditions (17). In fact, we show that the only solution to this mixed problem that disappears in a finite time is the null solution.

Theorem 2. Assume that the main hypotheses (a)-(c) are satisfied. If $t_{1} \geq 0$ is a finite time and $\left(u_{i}, \phi_{i j}, \theta, \varphi\right)$ is a solution of the mixed problem $\mathcal{P}$, so that $u_{i}(t)=0, \phi_{i j}(t)=0, \theta(t)=0, \varphi(t)=0$, for any $t \geq t_{1}$, then we have $u_{i}(t)=0, \phi_{i j}(t)=0, \theta(t)=0, \varphi(t)=0$, for any $t \geq t_{1}$.

Proof. To obtain the conclusion of Theorem 2, we consider the mixed problem $\mathcal{P}$ in the case of homogeneous boundary conditions and considering the initial null data

$$
\begin{gathered}
u_{i}\left(t_{1}, x\right)=0, \dot{u}_{i}\left(t_{1}, x\right)=0, \phi_{i j}\left(t_{1}, x\right)=0, \dot{\phi}_{i j}\left(t_{1}, x\right)=0, \\
\theta\left(t_{1}, x\right)=0, \varphi\left(t_{1}, x\right)=0, \dot{\varphi}\left(t_{1}, x\right)=0, t_{1} \geq 0, x \in D .
\end{gathered}
$$

If we take into account these null initial data, according to Theorem 1, the problem $\mathcal{P}$ admits only the null solution.

\section{Conclusions}

This paper is dedicated to the linear mixed initial-boundary value problem in the context of the theory of thermoelasticity for bodies with voids that have a dipolar structure. After we put down some auxiliary identities, we approach the issue of the uniqueness for the solution for the mixed problem. Then, we approach the question of the possibility of locating in time of solution for this problem, namely, we have shown that it is impossible to locate in a finite time of the solution of mixed problem. 
Even if we combined the effect of the dipolar structure with the effect of the voids and with the thermal one, not were ensured the sufficient conditions for deformations vanish in a finite time.

If we take into account the fact that the porous media with a dipolar structure very accurately models the structure and behavior of the bones, we can deduce that a concrete application of our calculations is the following. If a bone is hit in one part accidentally, the time interval after which the pain caused by the blow completely disappears cannot be established exactly. However, the porous bodies with a dipolar structure also model other practical media, such as polystyrene, artificial porous materials, rocks, soils, and so on. A number of concrete applications of the theoretical results from our study can be made for these particular media. To this aim we must consider the isotropic case because in this situation the number of coefficients decreases significantly. Then, we will look for a specialized laboratory, of high performance, that can provide us with concrete values of the coefficients, for specific materials.

Author Contributions: All four authors conceived the framework and structured the whole manuscript, checked the results, and completed the revision of the paper. The authors have equally contributed to the elaboration of this manuscript. All authors have read and approved the final form of the manuscript.

Funding: This research received no external funding.

Conflicts of Interest: The authors declare no conflicts of interest.

\section{References}

1. Eringen, A.C. Theory of thermo-microstretch elastic solids. Int. J. Eng. Sci. 1990, 28, 1291-1301. [CrossRef]

2. Eringen, A.C. Microcontinuum Field Theories; Springer: New York, NY, USA, 1999.

3. Marin, M.; Nicaise, S. Existence and stability results for thermoelastic dipolar bodies with double porosity. Contin. Mech. Therm. 2016, 28, 1645-1657. [CrossRef]

4. Marin, M.; Ellahi, R.; Chirila, A. On solutions of Saint-Venant's problem for elastic dipolar bodies with voids. Carpathian J. Math. 2017, 33, 219-232.

5. Mindlin, R.D. Micro-structure in linear elasticity. Arch. Ration. Mech. Anal. 1964, 16, 51-78. [CrossRef]

6. Green, A.E.; Rivlin, R.S. Multipolar continuum mechanics. Arch. Ration. Mech. Anal. 1964, 17, $113-147$. [CrossRef]

7. Fried, E.; Gurtin, M.E. Thermomechanics of the interface between a body and its environment. Contin. Mech. Therm. 2007, 19, 253-271. [CrossRef]

8. Nunziato, J.W.; Cowin, S.C. A nonlinear theory of materials with voids. Arch. Ration. Mech. Anal. 1979, 72, 175-201. [CrossRef]

9. Cowin, S.C.; Nunziato, J.W. Linear elastic materials with voids. J. Elast. 1983, 13, 125-147. [CrossRef]

10. Goodman, M.A.; Cowin, S.C. A continuum theory of granular material. Arch. Ration. Mech. Anal. 1971, 44, 249-266. [CrossRef]

11. Marin, M.; Baleanu, D.; Vlase, S. Effect of microtemperatures for micropolar thermoelastic bodies. Struct. Eng. Mech. 2017, 61, 381-387. [CrossRef]

12. Abbas, I.A. A GN model based upon two-temperature generalized thermoelastic theory in an unbounded medium with a spherical cavity. Appl. Math. Comput. 2014, 245, 108-115. [CrossRef]

13. Marin, M.; Florea, O. On temporal behaviour of solutions in thermoelasticity of porous micropolar bodies. An. St. Univ. Ovidius Constanta 2014, 22, 169-188. [CrossRef]

14. Othman, M.I.A.; Hasona, W.M.; Abd-Elaziz, E.M. Effect of Rotation on Micropolar Generalized Thermoelasticity with Two-Temperatures using a Dual-Phase-Lag Model. Can. J. Phys. 2014, 92, 149-158. [CrossRef]

15. Marin, M. Lagrange identity method for microstretch thermoelastic materials. J. Math. Anal. Appl. 2010, 363, 275-286. [CrossRef]

16. Marin, M.; Craciun, E.M. Uniqueness results for a boundary value problem in dipolar thermoelasticity to model composite materials. Compos. Part B Eng. 2017, 126, 27-37. [CrossRef]

17. Serrin, J. The Initial Value Problem for the Navier-Stokes Equations. In Nonlinear Problems; University of Wisconsin Press: Madison, WI, USA, 1963; pp. 69-98. 
18. Ciarletta, M.; Scalia, A. Some Results in Linear Theory of Thermomicrostretch Elastic Solids. Meccanica 2004, 39, 191-206. [CrossRef]

19. Knops, R.J.; Payne, L.E. On the Stability of Solutions of the Navier-Stokes Equations Backward in Time. Arch. Ration. Mech. Anal. 1968, 29, 331-335. [CrossRef]

20. Galdi, G.P.; Straughan, B. Stability of Solutions of the Navier-Stokes Equations Backward in Time. Arch. Ration. Mech. Anal. 1988, 101, 107-114. [CrossRef]

21. Payne, L.; Straughan, B. Improperly Posed and Nonstandard Problems for Parabolic Partial Differential Equations. In Elasticity: Mathematical Methods and Applications; Eason, R.W.O.G., Ed.; Ellis Horwood: Devon, UK, 1990; pp. 273-300.

22. Ames, K.A.; Payne, L.E. Stabilizing Solutions of the Equations of Dynamical Linear Thermoelasticity Backward in Time. Stab. Appl. Anal. Contin. Media 1991, 1, 243-260.

23. Ciarletta, M. On the Uniqueness and Continuous Dependence of Solutions in Dyna-mical Thermoelasticity Backward in Time. J. Therm. Streses 2002, 25, 969-984. [CrossRef]

24. Ciarletta, M.; Chirita, S. Spatial Behavior in Linear Thermoelasticity Backward in Time. In Proceedings of the Fourth International Congress on Thermal Stresses, Osaka, Japan, 8-11 June 2001; Chao, C.K., Lin, C.Y., Eds.; pp. 485-488,

25. Quintanilla, R. Impossibility of Localization in Linear Thermoelasticity with Voids. Mech. Res. Commun. 2007, 34, 522-527. [CrossRef]

26. Green, A.E.; Naghdi, P.M. On Undamped Heat Waves in an Elastic Solid. J. Therm. Stress. 1992, 15, $253-264$. [CrossRef]

27. Green, A.E.; Naghdi, P.M. Thermoelasticity without Energy Dissipation. J. Elast. 1993, 31, 189-208. [CrossRef]

28. Iovane, G.; Passarella, F. Saint-Venant's Principle in Dynamic Porous Thermoelastic Media with Memory for Heat Flux. J. Therm Stress. 2004, 27, 983-999. [CrossRef]

29. Passarella, F.; Tibullo, V. Some Results in Linear Theory of Thermoelasticity Backward in Time for Microstretch Materials. J. Therm. Stress. 2010, 33, 559-576. [CrossRef]

30. Chirila, A.; Marin, M. The theory of generalized thermoelasticity with fractional order strain for dipolar materials with double porosity. J. Mater. Sci. 2018, 53, 3470-3482. [CrossRef]

31. Marin, M.; Agarwal, R.P.; Codarcea, L. A mathematical model for three-phase-lag dipolar thermoelastic bodies. J. Ineq. Appl. 2017, 2017, 109. [CrossRef]

32. Quintanilla, R.; Straughan, B. Energy Bounds for Some Non-standard Problems in Thermoelasticity. Proc. R. Soc. Lond. A 2005, 461, 1-15. [CrossRef]

(C) 2020 by the authors. Licensee MDPI, Basel, Switzerland. This article is an open access article distributed under the terms and conditions of the Creative Commons Attribution (CC BY) license (http://creativecommons.org/licenses/by/4.0/). 